\title{
Frequency of Endocrinopathies and Characteristics of Affected Dogs and Cats in Southern Brazil (2004-2014)
}

\author{
Álan Gomes Pöppl', Isadora Comparsi Coelho², Camila Alves da Silveira², \\ Maurício Bianchini Moresco ${ }^{2}$, Guilherme Luiz Carvalho de Carvalho ${ }^{3}$
}

\begin{abstract}
Background: The increase in the life expectancy of dogs and cats in recent decades has heightened the incidence of aging-associated diseases. Among such diseases, endocrinopathies, such as obesity, stand out. In addition, there exists a lack of professionals with expertise in this area. Therefore, proper knowledge of the characteristics of patients affected by endocrinopathies as well as the frequency of these diseases aid in the recognition of endocrine syndromes in the general population. Thus, the aim of this study was to conduct a retrospective analysis of medical charts of dogs and cats compiled by a Division of Endocrinology in southern Brazil during a 10-year period.

Materials, Methods \& Results: An epidemiological survey of the medical charts of 1,400 dogs and cats compiled by a Division of Endocrinology between 2004 and 2014 was performed. The diagnostic data based on laboratory and hormone tests and on ultrasound scans were used as gold standard. The data were tabulated using Excel for Windows for the stratified analysis of the diagnoses. The major diseases (93.4\% of the cases) detected in dogs were hyperadrenocorticism (37\%), diabetes mellitus (22\%), hypothyroidism (11\%), and overweight/obesity (8\%), whereas the main diseases $(6.6 \%$ of the cases) detected in cats were diabetes mellitus (42\%) and hyperthyroidism (23\%). Some rare and uncommon diseases were diagnosed, such as hypoparathyroidism, pheochromocytoma, pituitary dwarfism, and diabetes insipidus, but they were present in less than $1 \%$ of the patients. Multiple endocrinopathies accounted for $8 \%$ of the cases, and the most frequent association was that between diabetes mellitus and hyperadrenocorticism in dogs and cats. The mean age of dogs was $9.11 \pm 3.43$ years, and $69 \%$ of the patients were female. The mean age of cats was $10.97 \pm 4.29$ years, and $58 \%$ of the patients were male.

Discussion: The high prevalence of endocrinopathies among female dogs is closely related to the higher prevalence of diabetes mellitus and hyperadrenocorticism in female patients, and these two diseases together accounted for 59\% of the cases. Among cats, the high prevalence of endocrinopathies among male cats was influenced by the large number of cases of diabetes mellitus, a disease that is typically more prevalent among tomcats. Some findings were alarming as they indicate probable lack of early diagnoses of some conditions (e.g., the prevalence of dogs with hyperadrenocorticism and diabetes three times greater than that reported by other countries), absence of patients younger than 10 years with hyperthyroidism, or failure to seek veterinary care for obese cats with initial presentation of overweight. Raising the awareness of tutors about the consequences of overweight can drastically reduce the incidence of diabetes mellitus in cats. Finally, knowledge about the characteristics of patients with the most common types of endocrinopathies is useful to clinicians as it can increase the predictive values of clinical and laboratory findings and help them include certain endocrinopathies as differential diagnoses for patients with some risk factors (age or sex). Anyway, the endocrinopathies, as well as the characteristics of the affected population in southern Brazil, are in line with similar studies on the most frequent diseases in other regions, taking into account small differences in breed and age distribution.
\end{abstract}

Keywords: adrenal diseases, endocrine pancreas, thyroid gland, obesity, epidemiology. 


\section{INTRODUCTION}

The increase in the life expectancy of dogs and cats as a result of technological breakthroughs in veterinary medicine, as well as of important changes in the relationship between tutors and pets in the past two decades, has been linked to a rise in the incidence of agingassociated diseases, such as oncological and endocrine disorders [5]. Accordingly, the prevalence of obesity among pets has heightened worldwide [4]. As adipose tissue has endocrine functions and as the large production of adipocytokines in obesity is associated with a broad array of health complications, obesity is seen nowadays as an endocrine and metabolic disease [17]. In addition, several endocrine diseases occur concomitantly with overweight/obesity [hyperadrenocorticism (HAC), hypothyroidism (HpoT), acromegaly, insulinoma], whereas other endocrine diseases, such as diabetes mellitus (DM), can actually be secondary to obesity $[5,6]$.

The description of highly prevalent endocrinopathies allows for better preparation of health professionals in the teaching setting, since there exists a paucity of clinical veterinarians who work with this specialty, which accounts for around $10 \%$ of the total number of veterinary visits for dogs and cats [14]. Owing to the lack of epidemiological data on endocrinopathies in Brazil, the aim of this study was to conduct an epidemiological survey and construct a profile of the small animals treated at an endocrinology outpatient clinic.

\section{MATERIALS AND METHODS}

A retrospective analysis was conducted to collect data on endocrinopathies and metabolic diseases in dogs and cats that had been gathered by a special Division of Veterinary Endocrinology in Porto Alegre, State of Rio Grande do Sul. The Division had collected data from several private clinics and from the Teaching Hospital of Universidade Federal do Rio Grande do Sul, and also from affiliated itinerant veterinarians during their visits to nearby towns. The information on each clinical case included species, breed, sex, age, and diagnosis. The patient's age at the time of admission was recorded, expressed as mean \pm standard deviation. The breed informed in the medical chart by the attending veterinarian was used as gold standard. The diagnoses were those recorded and confirmed by laboratory tests, hormone tests, and ultrasound scans. The data were tabulated using Excel for Windows for the stratified analysis of the diagnosis.

\section{RESULTS}

The Division of Veterinary Endocrinology attended to patients from towns located in different regions of the State of Rio Grande do Sul, including Pelotas (south), Alegrete, Uruguaiana, Rosário, São Gabriel, Bagé (grasslands region), Santa Maria, Cachoeira do Sul, Santa Cruz do Sul, Barros Cassal (central region), Ijuí, Passo Fundo, Lagoa Vermelha, Erechim (north), Caxias do Sul, Gramado, Bento Gonçalves, Farroupilha, Garibaldi (mountain region), Novo Hamburgo, Canoas, Esteio, São Leopoldo, Gravataí, Guaíba, Viamão, Alvorada (metropolitan area), and Capão da Canoa, Arroio do Sal, Torres (coastal region). Patients had also been brought in from Arroio do Silva, Imbituba, Criciúma, Florianópolis (State of Santa Catarina) and from Montevideo (Uruguay). Figure 1 shows the distribution of cities/towns of origin of the patients.

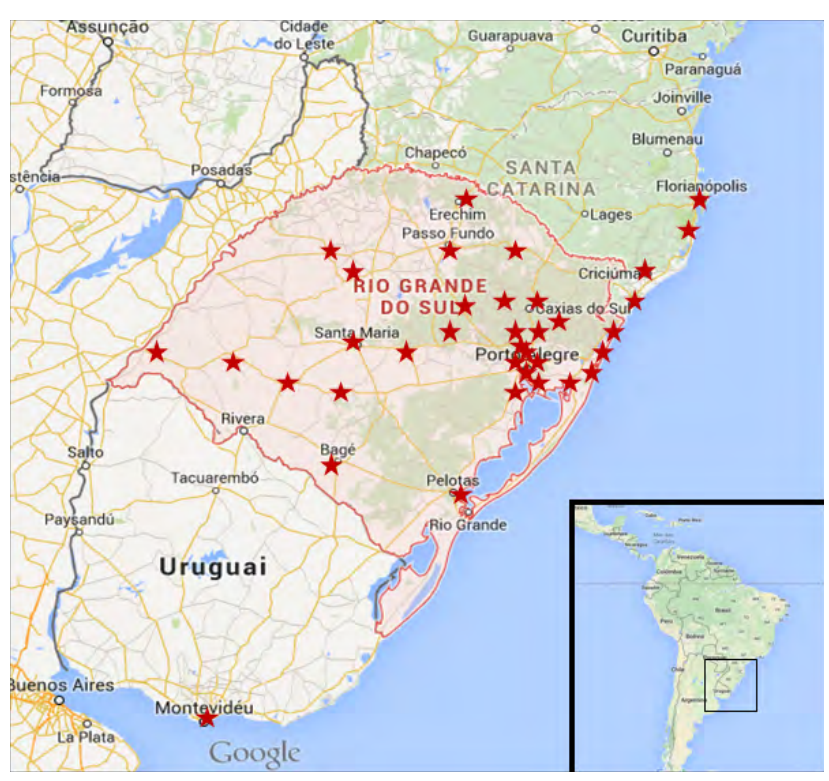

Figure 1. Distribution of cities/towns from which patients were brought in for veterinary care.

A total of 1,400 medical charts were assessed for the period between December 2004 and December 2014, including 1,308 dogs $(93.4 \%)$ and 92 cats $(6.6 \%)$. The mean age was $9.11 \pm 3.43$ years for dogs, $69 \%$ of which were female, and $10.97 \pm 4.29$ years for cats, $58 \%$ of which were male. Figure 2 shows the histograms for age distribution of the patients.

The major diseases diagnosed in dogs had been HAC (523 cases, 37\%), DM (315 cases, 22\%), and HpoT (154 cases, 11\%), as shown in Figure 3. Some patients had presented with more than one of these diseases; 87 of them $(6.21 \%)$ had been diagnosed with HAC and DM, accounting for $16.6 \%$ of the patients 
with DM secondary to HAC. Other associations between these 3 diagnoses were DM and HpoT in 8 dogs ( $0.57 \%$ of the total, but $2.53 \%$ of the total number of diabetic patients) and primary HpoT and HAC in 7 dogs $(0.5 \%$ of the total).

Dogs with HAC showed a mean age of 10.2 \pm 2.86 . Figure 4 displays a comparative histogram

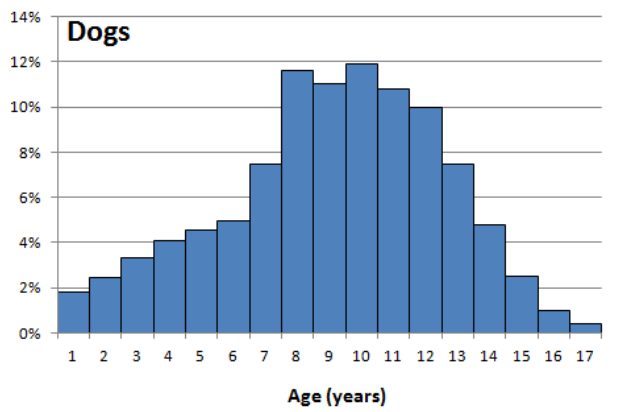

for the ages at diagnosis of patients with HAC, DM, HpoT and obesity. Sixty-nine percent of the patients with HAC were female and the most affected breeds were Poodle (35\%), mixed breeds (18\%), Dachshund (12\%), and Yorkshire (8\%). Table 1 shows the prevalence of the breeds diagnosed with HAC.

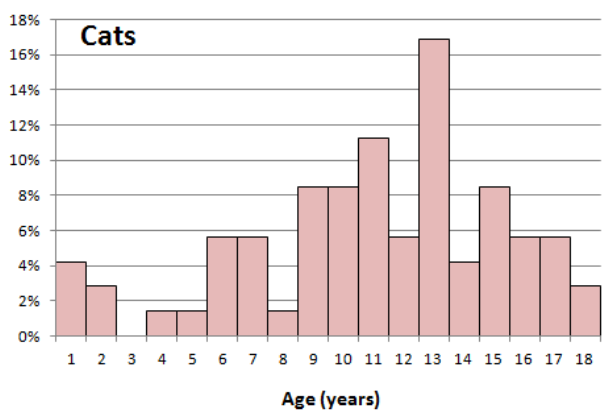

Figure 2. Histograms of age distribution of treated dogs and cats.
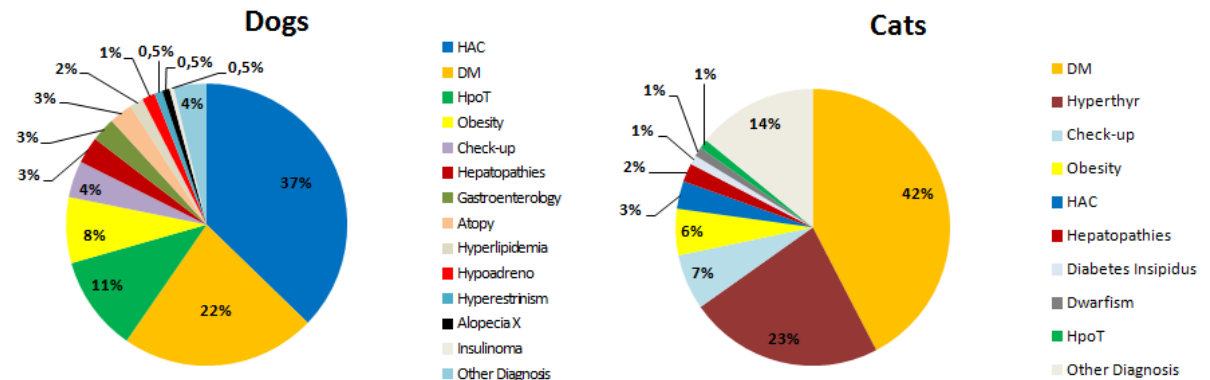

Figure 3. Distribution and frequency of diagnoses made in dogs and cats at the Division of Endocrinology between 2004 and 2014.

HAC

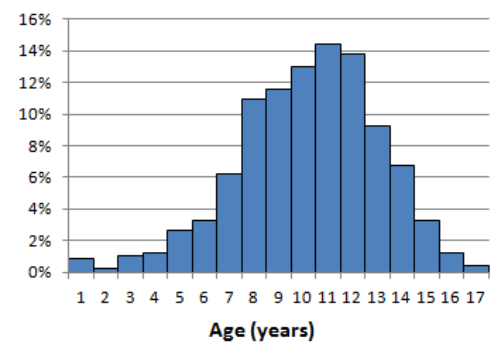

НpoT

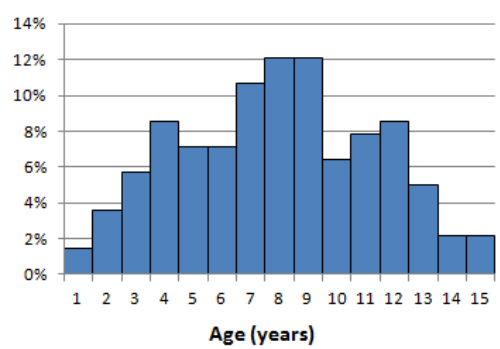

DM

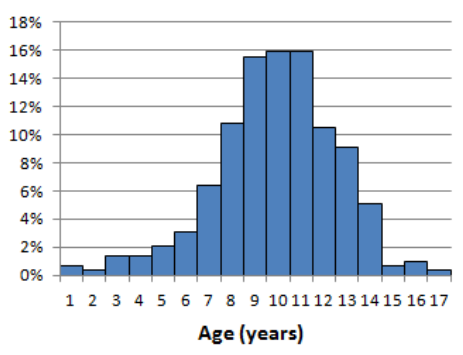

Obesity

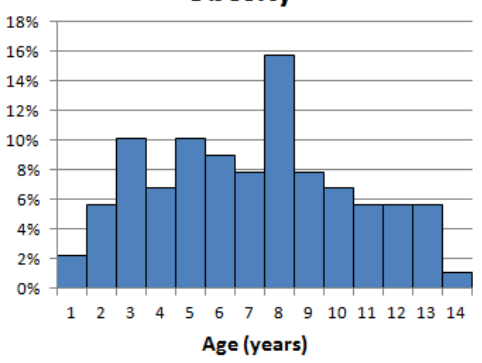

Figure 4. Comparative histogram of age at diagnosis of canine patients with HAC, DM, HpoT and obesity. 
Diabetic dogs showed a mean age of $9.9 \pm 2.65$ years. There was a female predominance $(72 \%$ of the cases) and Poodle (31\%), mixed-breed (20\%), Dachshund (8\%), Schnauzer (5\%), and Labrador (4\%) dogs were mostly often affected, followed by other breeds (32\%) as shown in Table 2.

HpoT was the third most common endocrinopathy among dogs, with a mean age of $8 \pm 3.39$ years and a female predominance $(62 \%)$. The following breeds were most frequently affected: mixed breeds $(24 \%)$, Beagle (13\%), Dachshund (11\%), Labrador (7\%), and Schnauzer (6\%). Table 3 shows the prevalence of the breeds diagnosed with HpoT.

Dogs with overweight/simple obesity (with no underlying endocrinopathy) accounted for $8 \%$ of the cases (106 patients). The patients' mean age was 9.22 \pm 3.46 years at admission. Among obese patients, only $20.7 \%$ were male. Table 4 describes the breeds most frequently diagnosed with overweight/obesity, showing that mixed-breed dogs were the most commonly affected (25.5\%), followed by Labrador (19.8\%), Poodle (10.4\%), Dachshund (8.5\%), and Beagle (4.7\%).

Curiously enough, many of the treated dogs had not been diagnosed with endocrinopathies, but with endocrine-like conditions that affected other systems, and had been brought in for regular checkups, as shown in Figure 3. Liver diseases had been the main findings in these cases (43 patients), in addition to clinical cases of chronic gastrointestinal diseases (39 patients), such as inflammatory bowel diseases, pancreatitis, and exocrine pancreatic insufficiency.

A negligible number of patients had been diagnosed with hyperestrinism (13 cases), alopecia X (12 cases), and insulinoma ( 7 cases). However, several other diagnoses had been made among the remain- ing 75 patients, including kidney diseases, urinary incontinence, epilepsy, skin diseases with different etiologies, corneal dystrophies, in addition to primary dyslipidemias, diagnosed in 22 cases (2\%). Other rare endocrinopathies, such as central diabetes insipidus (1 case), pheochromocytoma ( 2 cases), and primary hyperparathyroidism (1 case), were also identified, but diagnosed in an irrelevant number of cases in this series, representing uncommon endocrine diseases [5].

Among cats, diabetes mellitus (42\% of the cases) and hyperthyroidism ( $23 \%$ of the cases) were the major endocrinopathies (Figure 3). As also observed in dogs, several patients had been brought in for regular check-ups or for investigation of endocrine-like conditions such as chronic kidney disease, inflammatory bowel disease, psychogenic alopecia, or liver diseases. Only 5 cats had been treated for overweight/obesity. Some uncommon diagnoses were made in cats, such as pituitary dwarfism associated with diabetes insipidus (1 case), hypothyroidism (1 case), natural-occurring hyperadrenocorticism (3 cases), and iatrogenic hyperadrenocorticism (1 case). A concomitant case of hyperthyroidism and diabetes mellitus had been documented, and among the 3 cases of hyperadrenocorticism, two were also diabetic.

Diabetic cats showed a mean age of $10.9 \pm 4.29$ years, $72 \%$ of which were male. Mixed-breed (77\%) and Siamese $(15 \%)$ cats were the most frequently affected. Patients with hyperthyroidism showed a mean age of $14 \pm 1.27$ years, $29 \%$ of which were male, and mixed-breed cats accounted for $62 \%$ of the cases, followed by Siamese (14\%), Persian (5\%), and Himalayan $(5 \%)$ cats. Figure 5 displays a comparative histogram for the ages at diagnosis of feline patients with DM and hyperthyroidism.
Feline DM

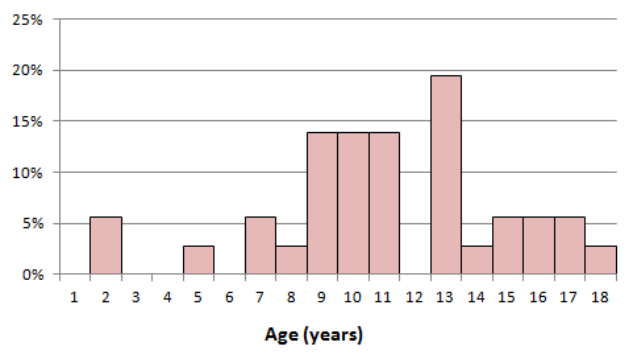

Feline Hyperthyroidism

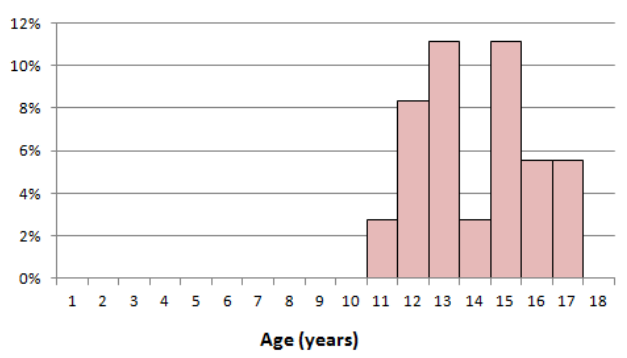

Figure 5. Comparative histogram of age at diagnosis of feline patients with DM and hyperthyreoidism. 
Table 1. Frequency of breed distribution of dogs diagnosed with hyperadrenocorticism at the Division of Endocrinology between 2004 and 2014.

\begin{tabular}{ccc}
\hline Breed & $\mathrm{N}$ & $(\%)$ \\
\hline Poodle & 181 & 34.7 \\
Mixed breed & 96 & 18.4 \\
Dachshund & 62 & 11.9 \\
Yorkshire Terrier & 40 & 7.7 \\
Bichon Frise, Doberman Pinscher & 17 (each) & 3.3 \\
Maltese & 15 & 2.9 \\
Beagle & 14 & 2.7 \\
Schnauzer & 10 & 1.9 \\
Shih Tzu & 9 & 1.7 \\
Labrador Retriever, Lhasa Apso & 7 (each) & 1.3 \\
Boxer, Cocker Spaniel & 6 (each) & 1.1 \\
Scottish Terrier & 4 & 0.8 \\
Chow-Chow, Fox Terrier & 3 (each) & 0.6 \\
American Pit Bull Terrier, American Staffordshire Terrier, Border Collie, & & \\
Chihuahua, German Shepherd Dog, German Spitz, Rottweiler, West & 2 (each) & 0.4 \\
Highland White Terrier, & & \\
Akita, Australian Shepherd Dog, Boston Terrier, Dogo Argentino, Fila & 1 (each) & 0.2 \\
Brasileiro, French Bulldog, Golden Retriever, Pekinese, Pug, Weimaraner & & 100 \\
\hline Total & 522 &
\end{tabular}

Table 2. Frequency of breed distribution of dogs diagnosed with diabetes mellitus at the Division of Endocrinology between 2004 and 2014.

\begin{tabular}{ccc}
\hline Breed & $\mathrm{N}$ & $(\%)$ \\
\hline Poodle & 98 & 31.1 \\
Mixed breed & 72 & 22.9 \\
Dachshund & 24 & 7.6 \\
Schnauzer & 17 & 5.4 \\
Labrador Retriever & 14 & 4.4 \\
Maltese, Doberman Pinscher & 11 (each) & 3.5 \\
Fox Terrier, Rottweiler & 10 (each) & 3.2 \\
Beagle & 6 & 1.9 \\
Bichon Frise, Yorkshire Terrier & 5 (each) & 1.6 \\
Cocker Spaniel & 4 & 1.3 \\
Boxer, Scottish Terrier & 3 (each) & 1.0 \\
Basset-Hound, Chow-Chow, Lhasa Apso, Pug, Shih Tzu & 2 (each) & 0.6 \\
American Staffordshire Terrier, Brittany, Dalmatian, French Bulldog, Great & 1 (each) & 0.3 \\
Dane, German Shepherd Dog, Pointer, Siberian Husky, Weimaraner & & 100 \\
\hline Total & 315 &
\end{tabular}


Table 3. Frequency of breed distribution of dogs diagnosed with hypothyroidism at the Division of Endocrinology between 2004 and 2014.

\begin{tabular}{ccc}
\hline Breed & $\mathrm{N}$ & $(\%)$ \\
\hline Mixed breed & 39 & 24.4 \\
Beagle & 20 & 12.5 \\
Dachshund & 18 & 11.3 \\
Labrador Retriever & 11 & 6.9 \\
Schnauzer & 10 & 6.3 \\
Poodle & 9 & 5.6 \\
Cocker Spaniel & 6 & 3.8 \\
Rottweiler & 5 & 3.1 \\
Lhasa Apso & 4 & 2.5 \\
Bichon Frise, Golden Retriever, Maltese, Pug & 3 (each) & 1.9 \\
Boxer, Doberman, Fila Brasileiro, American Pit Bull Terrier, Weimaraner, & 2 (each) & 1.3 \\
Yorkshire Terrier & 1 (each) & 0.6 \\
Akita, American Staffordshire Terrier, Basset-Hound, Border Collie, Chow- & 160 \\
Chow, Dalmatian, Doberman Pinscher, English Bulldog, French Bulldog, & 100 \\
\hline Fox Terrier, German Sheperd Dog, Shiba Inu, Scottish Terrier, Shih Tzu & Total & \\
\hline
\end{tabular}

Table 4. Frequency of breed distribution of dogs diagnosed with overweight/obesity at the Division of Endocrinology between 2004 and 2014.

\begin{tabular}{ccc}
\hline Breed & N & $(\%)$ \\
\hline Mixed Breed & 24 & 20.8 \\
Labrador Retriever & 19 & 19.8 \\
Poodle & 11 & 11.5 \\
Dachshund & 9 & 9.4 \\
Beagle & 5 & 5.2 \\
Doberman Pinscher, Shih Tzu, Yorkshire Terrier & 4 (each) & 4.2 \\
Cocker Spaniel, Fox & 3 (each) & 3.1 \\
Bichon Frise, Dalmatian & 2 (each) & 2.1 \\
Basset-Hound, Border Collie, Bull Terrier, Golden Retriever, Lhasa Apso, Pug & 1 (each) & 1.0 \\
\hline Total & 96 & 100 \\
\hline
\end{tabular}

\section{DISCUSSION}

The high prevalence of endocrinopathies in female dogs is closely related to the higher prevalence of diabetes mellitus and hyperadrenocorticism among females [5], which, together, accounted for 59\% of the cases among dogs. Among cats, the high prevalence of endocrinopathies among males was influenced by the large number of patients with diabetes mellitus, a disease that is typically more prevalent among older tomcats [13] as described in this study.

The detection of $16.6 \%$ of dogs with concurrent $\mathrm{HAC}$ and DM is three times higher than the 5\% estimated in an American case series of more than
4,000 cases for patients with DM secondary to HAC [5]. Nevertheless, a study in Argentina found a 10.5\% incidence of DM in HAC patients during a four-month treatment-free follow-up [7]. These data seem to suggest that several patients with HAC are only diagnosed after the onset of DM which, because of its more drastic features, prompts tutors to seek veterinary care for their pets much earlier. The concurrent development of $\mathrm{DM}$ and HpoT in $0.57 \%$ of the total patient population ( $2.53 \%$ of diabetic patients) demonstrates the potential occurrence of autoimmune polyendocrinopathy, given the pathogenicity of both diseases in the canine species [5]. The concurrent diagnosis of primary HpoT and HAC in 7 patients was made based on low serum 
concentration of free $\mathrm{T} 4$ by dialysis and high TSH levels in dogs in different HAC diagnostic stages (years before HAC development or after the diagnosis and control of HAC), ruling out the possibility that HpoT is secondary to endogenous hypercortisolism [2]. A case series study [3] described 35 cases of multiple endocrinopathies in dogs after analyzing more than 13,000 patients treated at a teaching veterinary hospital between 1996 and 2009, accounting only for $0.3 \%$ of the population treated there over 14 years. However, the most common combinations were HAC + DM (20/35) and HpoT + DM (10/35), which is in line with our findings that these combinations are the major multiple endocrinopathies among dogs.

Concerning HAC patients in this study, the mean age (10.2 \pm 2.86 years) was higher than that observed in 2 epidemiological surveys $[10,14]$ conducted in the Brazilian State of São Paulo, which reveal respectively a mean age of 9 years (range between 4 and 14), and mean age of $8.9 \pm 2.5$ years. Anyway, the profile clearly shows that this disease affects mainly middle-aged and old $\operatorname{dogs}[2,5]$. The studies undertaken at State of São Paulo $[10,14]$ also revealed that Poodles, mixed breeds, and Dachshunds are the most affected dogs, with a higher prevalence among females (66\% and 65\%), respectively.

As to dogs diagnosed with DM, the results obtained in this study are consistent with those gathered for Londrina, Brazilian State of Paraná [16], indicating $77 \%$ of diabetes among female dogs with a mean age of 10 years and a higher prevalence among Poodles $(32 \%)$ and mixed-breed dogs $(31 \%)$, and also with the data gathered for Guarulhos, State of São Paulo [14], which indicated $79.3 \%$ of the cases among female, being Poodles (65.9\%) and mixed-breed female dogs $(20.7 \%)$ the most frequent breeds.

HpoT was widely diagnosed in all age groups, despite the tendency towards diagnosis among middleaged and old dogs (Figure 4). Even though the literature does not indicate sex preponderance with respect to hypothyroidism [11], we found it in $62 \%$ of the females, which is also corroborated by studies carried out State of São Paulo by other authors [1,14], who detected rates of $61.5 \%$ and $66 \%$, respectively. Regarding the most commonly affected breeds (Table 3 ), HpoT is probably the disease with the largest variation in prevalence across different breeds in Brazilian studies; Labrador, Cocker, Beagle, Pit Bull, and Dachshund are quite common $[1,14]$ according to our findings.
The demographic data in this study are in line with the results obtained by an European study [4] for a population predominantly comprised of female dogs with overweight/obesity, which also showed that excessive weight is a common finding in older animals. Notwithstanding, the present study detected $79.3 \%$ of overweight among female patients whereas the study above mentioned obtained 60\% [4]. As shown in Table 4, Labrador, Basset, Dachshund, and Beagle had been previously considered to be more susceptible to obesity, even though this type of bias is related to local preferences for some breeds [4].

A considerable percentage of dogs was referred to endocrinological assessment due to liver diseases (43 patients), in which cases both abnormal laboratory findings and occasional abdominal bulging were suggestive of hyperadrenocorticism [5]. A total of 38 patients (3\%) had presented with iatrogenic hyperadrenocorticism as a result of the chronic use of glucocorticoids for the treatment of atopic dermatitis [2]. However, a smaller number (21 cases, $2 \%$ ) had been diagnosed with hypoadrenocorticism, of which 16 were considered primary cases and 5 were iatrogenic cases secondary to the administration of mitotane or trilostane, used in the treatment of hyperadrenocorticism [5], an occasional complication of anti-HAC therapy. In spite of that and despite the fact that hypoadrenocorticism is regarded as a rare condition, the frequency of natural-occurring cases outnumbered that of iatrogenic ones.

Concerning the data obtained for the feline species, the mean age of diabetic patients was $10.9 \pm$ 4.29 years, and $72 \%$ of the patients were male, being thus consistent with epidemiological data on feline DM collected in other countries, such as USA, Australia, and European countries [13], according to which age older than 7 years and neutering of males are the major risk factors for the development of the disease [15]. Nonetheless, as far as breed susceptibility is concerned, only Burmese Australian cats are at a higher risk for DM [13,15], but as this breed is not popular in Brazil, it was not analyzed in this study. Obesity is another important risk factor for the development of feline DM [5], and curiously, less than 5\% of the patients retrospectively assessed in the present study had been taken to veterinary care due to overweight. This is an alarming finding as it may indicate pet owners' collusive behavior towards the problem [4]. This finding should step up awareness-raising campaigns about 
obesity as a medical problem that must be discussed among students, health professionals, and tutors, since it is associated not only with DM, but also with several other comorbidities [6].

In cats with hyperthyroidism, the high mean age at diagnosis (14 \pm 1.27 years $)$ is consistent with epidemiological data on feline hyperthyroidism that show that more than $90 \%$ of the cases are older than 10 years at diagnosis [5]. However, the fact that no patient was diagnosed with hyperthyroidism before the age of 10 years could actually reveal shortcomings in the diagnostic and investigation processes in the region, combined with the failure to observe early diagnoses and the report of only the most severe cases and, consequently, the referral of only the most classic cases to veterinary care. In this case series, mixed-breed cats were the most frequently affected (62\%), confirming the finding that hyperthyroidism is more frequent among these animals than among purebred ones [12]. Even though Siamese and Himalayan cats are at a lower risk for hyperthyroidism, both breeds were assessed in this study; however, it was not possible to confirm whether they were actually purebreds. Interestingly, although hyperthyroidism has no sex predilection [12], $71 \%$ of the patients with hyperthyroidism were female.

As cats accounted for quite a low percentage of patients in this study, the occurrence of uncommon diseases was underreported. However, the growing association between diabetes mellitus and acromegaly in cats could not be confirmed in any of the cases studied herein. The difficulties in the diagnosis of acromegaly [9], based on pituitary CT and magnetic resonance and on the serum concentration of insulin-like growth factor 1 (IGF-1), explain this poor association, especially because these techniques have been available in the region for only some years and at prohibitive costs. Nevertheless, the role of other endocrine diseases that lead to DM in felines (DM type III) may be observed in the associations between DM + hyperthyroidism and $\mathrm{DM}+\mathrm{HAC}$ in the cats described herein [8].

\section{CONCLUSION}

The endocrinopathies, as well as the characteristics of the affected population in southern Brazil, are in line with similar studies on the most frequent diseases in other regions, taking into account small differences in breed and age distribution. Furthermore, the data may partly indicate shortcomings in the diagnosis of common clinical pictures, such as hyperadrenocorticism in dogs and obesity, hyperthyroidism, and acromegaly/hypersomatotropism in cats. The description and knowledge of the characteristics of patients affected by the most common endocrinopathies in the routine care of dogs and cats are useful to clinicians in two ways. First, they aid in the assessment of positive predictive value and/or negative predictive value of some clinical and laboratory findings during the treatment of patients with suspected endocrinopathies. Second, when treating a patient with a risk factor (sex or age) for a given endocrine disease, the possibility of such endocrinopathy should be included in the differential diagnoses considering its initial presentation in dogs or cats.

Acknowledgements. We are thankful to all staff from the Service of Statistic and Medical Archive of the Hospital de Clínicas Veterinárias da UFRGS for support throughout the survey of the medical charts.

Declaration of interest. The authors report no conflicts of interest. The authors alone are responsible for the content in writing of the paper.

\section{REFERENCES}

1 Aoki C.G., Palacios Junior R.J., Rondelli M.C.H., Sobreira M.F.R. \& Costa M.T. 2012. Aspectos clínicos do hipotireodismo canino: estudo retrospectivo. In: Anais $2^{\circ}$ Congresso Internacional da ABEV. Revista Clínica Veterinária. Supl. (Búzios, Brasil). p.26.

2 Behrend E.N. \& Melian C. 2013. Hyperadrenocorticism in dogs. In: Rand J. (Ed). Clinical Endocrinology of Companion Animals. Ames: Wiley-Blackwell, pp.43-64.

3 Blois S.L., Dickie E., Kruth S.A. \& Allen D.G. 2011. Multiple endocrine diseases in dogs: 35 cases (1996-2009). Journal of the American Veterinary Medical Association. 238(12): 1616-1621.

4 Diez M. \& Nguyen P. 2006. The epidemiology of canine and feline obesity. Waltham Focus. 16(1): 2-8.

5 Feldman E.C., Nelson R.W., Reusch C., Scott-Moncrieff J.C. 2015. Canine and Feline Endocrinology. 4th edn. Saint Louis: Saunders, 688p.

6 German A.J. 2006. The Growing problem of obesity in dogs and cats. Journal of Nutrition. 136(Suppl 7): s1940-s1946. 
7 Miceli D.D., Gallelli M.F., Blatter M.F., Martiarena B., Brañas M.M., Ortemberg L.R., Gómez N.V. \& Castillo V.A. 2012. Low dose of insulin detemir controls glycaemia, insulinemia and prevents diabetes mellitus progression in the dog with pituitary-dependent hyperadrenocorticism. Research in Veterinary Science. 93(1): 114-120.

8 Nelson R.W. \& Reusch C.E. 2014. Classification and etiology of diabetes in dogs and cats. Journal of Endocrinology. 222(3): T1-T9.

9 Niessen S.J.M., Church D.B. \& Forcada Y. Hypersomatotropism, Acromegaly, and Hyperadrenocorticism and Feline Diabetes Mellitus. Veterinary Clinics of North America: Small Animal Practice. 43(2): 319-350.

10 Palacios Junior R.J., Aoki C.G., Rondelli M.C.H., Sobreira M.F.R. \& Costa M.T. 2012. Aspectos clínicos do hipercortisolismo espontâneo em 20 cães: estudo retrospectivo. In: Anais $2^{\circ}$ Congresso Internacional da ABEV. Revista Clínica Veterinária. Supl. (Búzios, Brasil). p.27.

11 Panciera D. 2013. Hypothyroidism in dogs. In: Clinical Endocrinology of Companion Animals. Ames: Wiley-Blackwell, pp.263-272.

12 Peterson M.E. 2013. Hyperthyroidism in cats. In: Clinical Endocrinology of Companion Animals. Ames: WileyBlackwell, pp.295-310.

13 Rand J. 2013. Feline diabetes mellitus. In: Clinical Endocrinology of Companion Animals. Ames: Wiley-Blackwell, pp.169-190.

14 Schnobel A.M., Cardone R., Martorelli C.R., Winkel V.M. \& Marco V. 2006. Aspectos epidemiológicos das endocrinopatias em cães atendidas no Hospital Veterinário da Universidade de Guarulhos (UNG) entre agosto 2004 à abril 2006. In: Anais $6^{\circ}$ Congresso Paulista de Clínicos Veterinários de Pequenos Animais. (São Paulo, Brasil). p.185.

15 Sparkes A.H., Cannon M., Church D., Fleeman L., Harvey A., Hoenig M., Peterson M.E., Reusch C.E., Taylor S. \& Rosenberg D. 2015. ISFM Consensus guidelines on the practical management of diabetes mellitus in cats. Journal of Feline Medicine and Surgery. 17(3): 235-250.

16 Zanutto M.S., Diogo C.C., Silva F.M.F., Flaiban K.K.M.C. \& Beloni S.N.E. 2012. Estudo retrospectivo de cães com diabetes melito (DM) atendidos no Hospital Veterinário da Universidade Estadual de Londrina (HV-UEL). In: Anais $2{ }^{\circ}$ Congresso Internacional da ABEV. Revista Clínica Veterinária. Supl. (Búzios, Brasil). p.22.

17 Zoran D.L. 2010. Obesity in dogs and cats: a metabolic and endocrine disorder. Veterinary Clinics of North America: Small Animal Practice. 40(2): 221-239. 